\title{
Model studies of air entrainment in the Muela drop shaft
}

Proc. Instn Civ. Engrs Wat., Marit. \& Energy, 1992, 96, Sept, 209-210

Paper 9686

\section{Dyson, Aeration Systems}

As I have investigated the air entraining properties of vertical drop shafts fed from a vortex chamber, I well appreciate the time the Authors spent assessing the results published by others, and the frustration felt when correlations across the whole range could not be established. Our direct work was with shafts $8 \mathrm{~m}$ long, and $50 \mathrm{~mm}$ and $100 \mathrm{~mm}$ in dia., and also with rectangular columns $4 \mathrm{~m}$ high.

50. In addition, results on vortex fed shafts $150 \mathrm{~mm}$ and $300 \mathrm{~mm}$ in dia., and about $3 \mathrm{~m}$ and $6 \mathrm{~m}$ in length were acquired, as was further information on $150 \mathrm{~mm}$ and $300 \mathrm{~mm}$ square shafts.

51. Data from our tests yielded the extreme values given in Table 2 , which, to aid comparison, are converted to scale ratios of the Muela tests.

52. Prototype predictions of minimum drop for entrainment to occur vary widely between scales and are clearly unreliable, although Mateo's comment that predictions based on $1 / 6$ scale tests are acceptable appears valid. In this case, a $1 / 6$ scale model cannot be considered a laboratory test rig.

53. It is generally recognized that the volume of air entrained depends on the velocity of the impinging jet, which must itself depend on the distance travelled down the shaft. The stage relationship shown for the tunnels indicates a hydraulic grade level in the shaft of 1749 for a flow rate of $50 \mathrm{~m}^{3} / \mathrm{s}$.

54. At this drop in the prototype of $9.5 \mathrm{~m}$, the jet velocity is expected to be $13 \mathrm{~m} / \mathrm{s}$, with total air volume ingested about $12 \mathrm{~m}^{3} / \mathrm{s}$ : i.e. an air - water ratio of about $25 \%$. Should this air be drawn down and the voids' ratio in the column reach $20 \%$, then the standing water level in the shaft would rise by about $3 \mathrm{~m}$. Should this occur, then the volume of air entrained would be expected to decrease, causing the water level to fall. This apparently occurred during the tests. Do the Authors consider that such surges would occur and, if so, what would be their likely magnitude?

Table 2

\begin{tabular}{c|c|c|c|c}
\hline Scale ratio & $1 / 16$ & $1 / 35$ & $1 / 50$ & $1 / 100$ \\
\hline Minimum drop & & & & \\
at minimum flow: $\mathrm{m}$ & $1 \cdot 6$ & 0.8 & 0.6 & 0.5 \\
Prototype equivalent: $\mathrm{m}$ & $25 \cdot 6$ & 29 & 30 & 50 \\
Maximum air -water: \% & 28 & 22 & 19 & 12 \\
\hline
\end{tabular}

55. The geology and topography of the site probably dictated the possible design options, but as an alternative to the long de-aeration chamber, would not a hydro-cyclone type separator have been more compact?

\section{J. Kenn, Fellow}

The Authors have properly recognized that air entrainment is not correctly scaled when model tests are undertaken using the criterion of Froude number similarity. For this reason, the Authors have resorted to the not uncommon practice of testing models at differing scales in an attempt to assess the magnitude of the errors necessarily introduced by the inadequate scaling of the dynamic processes. ${ }^{18-20}$

57. In dealing with flow-induced aeration, it is misleading from the outset merely to contemplate Froude numbers, or Reynolds numbers, or even Weber numbers, as scaling criteria.

58. The dominant forces which influence flow-induced aeration are the viscous forces and the surface tension forces which are acting in opposition. Under equilibrium conditions, these forces will be both equal and opposite. Accordingly, the resultant (or 'inertia') forces will be zero (or negligible) and the scaling criterion will (in effect) necessitate similarity of ratios of (Weber number/Reynolds number), i.e.

$$
\left(\frac{\rho \mathrm{v}^{2} L}{\sigma} / \frac{\rho \mathrm{v} L}{\mu}\right) \text { or, hence } \frac{\mu \mathrm{v}}{\sigma}
$$

Thus, with identical pairs of fluids, the use of full-scale velocity becomes the necessary criterion for flow similarity. ${ }^{21,22}$

59. The same criterion also applies to the converse situations when a water spray is induced by an air flow.

60. The needs and requisites for this type of model testing have also been clearly and graphically illustrated by Professor F. Vasco Costa. ${ }^{23}$

61. In the present Paper, the Authors have suggested that the use of full-scale velocities and pressures in model testing presents some difficulties.

62. Elsewhere, however, other tests-e.g. on air-entraining siphons of dissimilar geometries and dissimilar cross-sectional areas have been carried out successfully under fullscale velocity and full-scale pressure conditions, and have clearly demonstrated the feasibility and the usefulness of such tests. Indeed, very close correlations of air and water flows have been obtained between such tests. ${ }^{24}$
Paper published: Proc. Instn Civ. Engrs, Part 2, 1991, 91, Sept., $417-434$ 
63. In a somewhat different but nevertheless analogous and related context (concerned with cavitation), further tests with full-scale velocities and pressures have also demonstrated the feasibility and the merits of such a testing procedure. ${ }^{\mathbf{2 5 , 2 6}}$

64. In the long term, Professor Stephenson's research students could perhaps still profitably find useful fields of study by exploring certain aspects of the drop shaft problems when tested under full-scale velocities and pressures.

\section{Professor Stephenson and Mr Metcalf}

Mr Dyson has added some valuable data to the air entrainment tables that we produced. He indicates higher air entrainment ratios, which are most likely attributable to a different configuration. As indicated in the references in the Paper, the velocity of the impinging jet is the most critical factor with respect to air entrainment rate. However, it is not entirely dependent on the distance travelled down the drop shaft, as a terminal velocity can be reached quite soon owing to the upward flow rate of air on one side of the jet and the downward flow resistance on the other side. Mr Dyson does not indicate from where his air-water ratio of $25 \%$ is derived, but it must be borne in mind that the configuration was such that a large proportion of the air subsequently rose back up the drop shaft and was not entrained around the elbow.

66. The reason given for the surging from the front shaft to the drop shaft appears correct. When the drop was high, it would entrain a large volume of air which would reappear in the vent shaft; when this air pocket reached the surface of the vent shaft, it would displace that volume of water which would tend to flow relatively backwards towards the drop shaft, thereby drowning out the drop to an extent, and reducing air entrainment. To extrapolate to the prototype would, however, be difficult as it is highly dependent on the air pocket size, and this appears to enlarge as small bubbles are picked up going along the deaeration chamber.
67. A hydro-cyclone type separator was considered only in passing for the de-aeration. The length needed for the de-aeration chamber was all part of the tunnel anyway, and a hydrocyclone separator would have required a wider excavation in poor rock, thereby adding to the cost.

68. MrKenn further supports the idea that it is the water velocity which dictates the air entrainment characteristics. However, as mentioned in the Paper, hydraulic jumps may form where they would otherwise not occur if the velocity is the only consideration in scaling down. Hydraulic jump air entrainment occurs as a result of a roller formation, whereas the other air entrainment phenomena referred to by Mr Kenn are primarily surface tension related.

\section{References}

18. Hydraulics Research Station. Model investigation of a typical drop shaft and de-aeration chamber. Research Station, Wallingford, 1965, Report EX 264, DSIR.

19. Ford S. E. and Elliot S. G. Investigation and design of the Plover Cove Water Scheme. Proc. Instn Civ. Engrs, 1965, 32, Oct., 255-293; 1966, 35, Oct., 342-358.

20. Camichel C. and Escande L. Similitude hydrodynamique et technique des modèles réduits. $P u b$. Sc.et. Tech. du Min. de l'Air, 1938, No. 127.

21. KENN M. J. and ZANKER K. J. Aspects of similarity for air-entraining water flows. Nature, 1967, 213, 5071, Jan., 59-60.

22. KENN M. J. Dynamical similarity for flow systems in which inertia effects are small. J. Instn Wat. Engrs, 1969, 23, 4, June, 251-253.

23. Vasco Costa F. Time-scale selection in hydraulic modelling. J. Coastal Res., 1990, Special Issue, No 7, Spring, 29-40.

24. KenN, M. J. Further studies concerning the aircontrol of siphonic water flow. J. Instn Wat. Engrs, 1965, 19, No. 3, May, 231-238.

25. KenN M. J. and Garrod A. D. Cavitation damage and the Tarbela-tunnel collapse. Proc. Instn Civ. Engrs, Part 1, 1981, 70, Feb., 65-89; Discussion: 1981, 70, Nov., 799-810.

26. KENN M. J. Cavitation and cavitation damage in concrete structures. Proc. 6th Int. Conf. on Erosion by Liquid and Solid Impact, Cambridge, Sept. 1983, 12, 1-6 (Cavendish Laboratory, University of Cambridge). 